\title{
Cierre transcatéter de ductus arterioso persistente
}

\author{
ALEJANDRO ÁLVAREZ J. ${ }^{1}$, VANESSA CARRILLO R. ${ }^{2}$, \\ EUGENIO SANHUEZA H. ${ }^{3}$, RODRIGO NAZAL L. ${ }^{4}$
}

1. Pediatra, Cardiólogo Infantil. Servicio de Pediatría Hospital Las Higueras Talcahuano. Departamento de Pediatría, Facultad de Medicina Universidad de Concepción.

2. Becada de Pediatría. Servicio de Pediatría Hospital Las Higueras Talcahuano. Departamento de Pediatría, Facultad de Medicina Universidad de Concepción.

3. Médico Anestesiólogo Cardiovascular, Laboratorio de Hemodinamia, Hospital Las Higueras Talcahuano.

4. Pediatra, Cardiólogo Infantil. Servicio de Pediatría Hospital Las Higueras Talcahuano.

\begin{abstract}
Transcatheter closure of patent ductus arteriosus

Percutaneous closure of patent ductus arteriosus (PDA) is a well-known technique and is the first choice for this condition. Objective: To report our pioneer experience in the region, the first 6 percutaneously treated PDA cases. The smallest diameter was less than $3 \mathrm{~mm}$ and they were closed using transcatheter technique with controlled-release coils in Las Higueras Hospital, Talcahuano. Patients and Methods: Between December, 2010 and September, 2011, six female patients carrying PDA were treated via interventional catheterization. Three presented a type A1 from Krichenko ductus arteriosus, two patients of the type A2 and one patient of the type E. Under general anesthesia, cardiac catheterization was performed via the femoral artery and aortography. The ductus was reached using a multipurpose catheter and through this, the coil was inserted closing the abnormality from the pulmonary to the aortic end using the controlled-release coil. Results: All patients achieved angiographic ductal closure without residual shunt before discharge. The procedure lasted an average of 55 minutes, the mean fluoroscopy time was 14 minutes and there were no complications. Patients were discharged the day after surgery after undergoing chest radiography and echocardiography. In later follow-ups, patients were asymptomatic. Conclusion: The closure of percutaneous PDAs is an effective, safe and less invasive technique, which has been performed for some time in Santiago, with this being the first regional report. (Key words: Patent ductus arteriosus, transcatheter closure, coils).

Rev Chil Pediatr 2012; 83 (6): 577-581
\end{abstract}

\section{RESUMEN}

El cierre percutáneo del ductus arterioso persistente (DAP) es una técnica bien establecida y es la primera elección en esta patología. Objetivo: Comunicar nuestra experiencia pionera en la región, de los primeros 6 casos tratados percutaneamente de DAP cuyo diámetro menor fue inferior a $3 \mathrm{~mm}$, cerrados con técnica transcatéter con coil de liberación controlada. Pacientes y Método: Desde diciembre de 2010 a septiembre de 2011 se

Recibido el 02de febrero de 2012, devuelto para corregir el 09 de abril de 2012, segunda versión el 18 de junio de 2012, tercera versión el 08 de agosto de 2012, aceptado para publicación el 23 de agosto de 2012.

Correspondencia a:

Dr. Alejandro Álvarez J.

E-mail: alalvare@udec.cl 
han intervenido en el Hospital Las Higueras de Talcahuano con cateterismo intervencional 6 pacientes de sexo femenino portadoras de DAP. Tres eran portadoras de un ductus Krichenko A1, dos ductus de tipo Krichenko A2 y uno de tipo E. Bajo anestesia general, se efectuó sondeo cardíaco vía arteria femoral y aortografía. Con un catéter multipropósito se canalizó el ductus y a través de este se avanzó el coil, efectuando el cierre del defecto desde extremo pulmonar al aórtico con el coil de liberación controlada $\left(\mathrm{COOK}^{\mathrm{R}}\right)$. Resultados: En todos los pacientes se logró cierre angiográfico ductal, sin shunt residual a la ecocardiografía previo al alta. El procedimiento duró un promedio de 55 minutos, el tiempo de fluoroscopia promedio fue de 14 minutos y no se registraron complicaciones, los pacientes fueron dados de alta al día siguiente de la intervención, post radiografía tórax y ecocardiograma. En los controles posteriores las niñas se encuentran asintomáticas, con dispositivo in sede a los controles ecocardiográficos. Conclusión: El cierre de DAP por vía percutánea es una técnica efectiva, segura y menos invasiva, realizada hace algún tiempo en nuestro país en la capital, siendo este el primer reporte regional.

(Palabras clave: Ductus arterioso persistente, cierre transcatéter, coils).

Rev Chil Pediatr 2012; 83 (6): 577-581

\section{Introducción}

El ductus arterioso persistente (DAP) conecta el tronco de la arteria pulmonar cerca del origen de su rama izquierda con la aorta descendente y se cierra espontáneo en el período neonatal. Cuando persiste más allá de ese período se denomina ductus arterioso persistente. Siendo una cardiopatia congenita con cortocircuito de izquierda a derecha y según la magnitud de este cortocircuito, será la presencia de insuficiencia cardíaca o desarrollar enfermedad vascular pulmonar irreversible si no es resuelto en forma oportuna.

Según series históricas la incidencia de DAP aislado en niños nacidos a término es de 1 en cada 2 000-2 500 recién nacidos vivos, representando el 5 a $10 \%$ de todas las cardiopatías congénitas. Es más frecuente en niñas $(2: 1)$ respecto a niños ${ }^{1}$.

La técnica del cierre percutáneo fue descrita por primera vez por Porstmann en el año 1967 sufriendo variaciones a través de los años con el fin de disminuir los costos y complicaciones. En el año 1992 Cambier describe el cierre de DAP utilizando espirales liberados a través de catéteres introducidos por vía femoral.

En los grupos cardioquirúrgicos desarrollados el DAP es actualmente tratado mediante cierre percutáneo siendo ésta la primera elección en la mayoría de los pacientes con peso superior a los $4,5 \mathrm{~kg}$, quedando el cierre quirúrgico reservado principalmente para los prematuros, DAP asociados a otras cardiopatias congenitas que deben ser tratadas quirúrgicamente, paciente con imposibilidad del acceso vascular tanto venoso como arterial y DAP de mala anatomía, ya que no son candidatos a cierre percutáneo ${ }^{2-3}$. En Chile, este procedimiento se realiza principalmente en centros de la Región Metropolitana que funcionan como centros de referencia. Este trabajo tiene como objetivo mostrar nuestra experiencia en los primeros casos de cierre percutaneo del DAP en el Hospital Las Higueras Talcahuano.

\section{Pacientes y Método}

Desde diciembre de 2010 hasta junio de 2011, 6 pacientes con diagnóstico clínico y ecocardiográfico DAP fueron sometidos a cierre percutáneo con dispositivo coil $\mathrm{COOK}^{\mathrm{R}}$ de liberación controlada, todos eran de sexo femenino. En todas se realizó ECG, radiografia y ecocardiograma además de exámenes de laboratorio hemograma, creatininemia, tiempo protrombina y glicemia previo al procedimento. Se obtuvo el consentimiento informado de los padres en el cual se explicaba el procedimiento en términos comprensibles para ellos además de mencionar los posibles riesgo, complicaciones y beneficios de efectuar este tratamiento. Los criterios de inclusión fueron: peso mayor de $4 \mathrm{~kg}$, resistencias vasculares pulmonares $<8$ Unidades Wood $/ \mathrm{m}^{2}$, y el tamaño menor del ductus $\geq 1,5 \mathrm{~mm}$. Las pacientes fueron enviadas para efectuar cierre del DAP 
por presentar cuadros bronquiales a repetición e insuficiencia cardíaca.

\section{Procedimiento}

El procedimiento se realizó en el laboratorio de hemodinamia. Bajo anestesia general se efectuó punción en arteria femoral derecha o izquierda y se procedió a colocar un introductor 4Fr o 5Fr según el peso del paciente, a través del cual se efectúa aortografía con un catéter Pig Tail para visualizar la anatomía (clasificación de Kirchenko ${ }^{4}$ ) y el tamaño angiográfico del DAP (figura 1). Con la información anterior se elije el dispositivo a utilizar siendo este 1,5 a 2 veces mayor al diámetro menor del defecto. Posteriormente, por medio de un catéter multipropósito y con una guía hidrofílica se canaliza el DAP colocando el catéter en arteria pulmonar para realizar medida de la presión en el territorio pulmonar. Luego a través del catéter multipropósito se introduce el dispositivo con su mecanismo de desprendimiento de la espiral, dejando 1 a 11/2 vueltas del coil en la arteria pulmonar y avanzar despacio retrógradamente la guía de liberación para formar el resto de las vueltas en la ampolla aórtica, después se procede a constatar la estabilidad del dispositivo para su liberación (figura 2). Transcurrido $10 \mathrm{~min}$ se realizó aortografía de control para verificar que el conducto ha quedado ocluido. Los pacientes reciben una dosis de heparina 50-100 U por kilo después de efectuada la aortografía para caracterizar el DAP y profilaxis con cefazolina una dosis intraoperatoria y una segunda a las $8 \mathrm{~h}$ post procedimiento. Todas las pacientes se dieron de alta al día siguiente previo a la toma de radiografía de tórax y ecocardiograma de

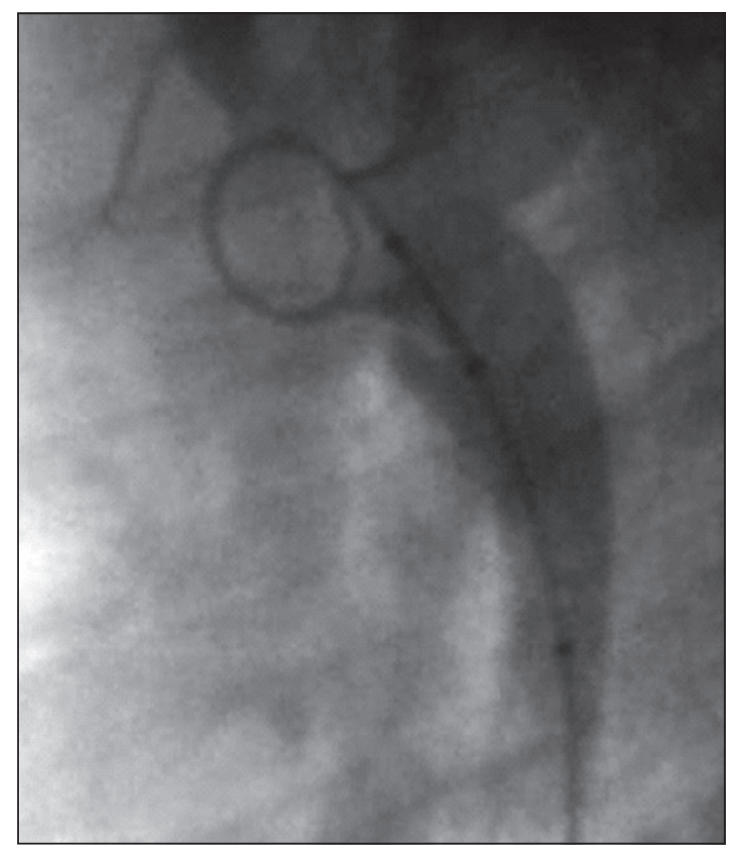

Figura 1. Aortografía en visión lateral donde se aprecia imagen de ductus arterioso persistente de diámetro mínimo $2 \mathrm{~mm}$.

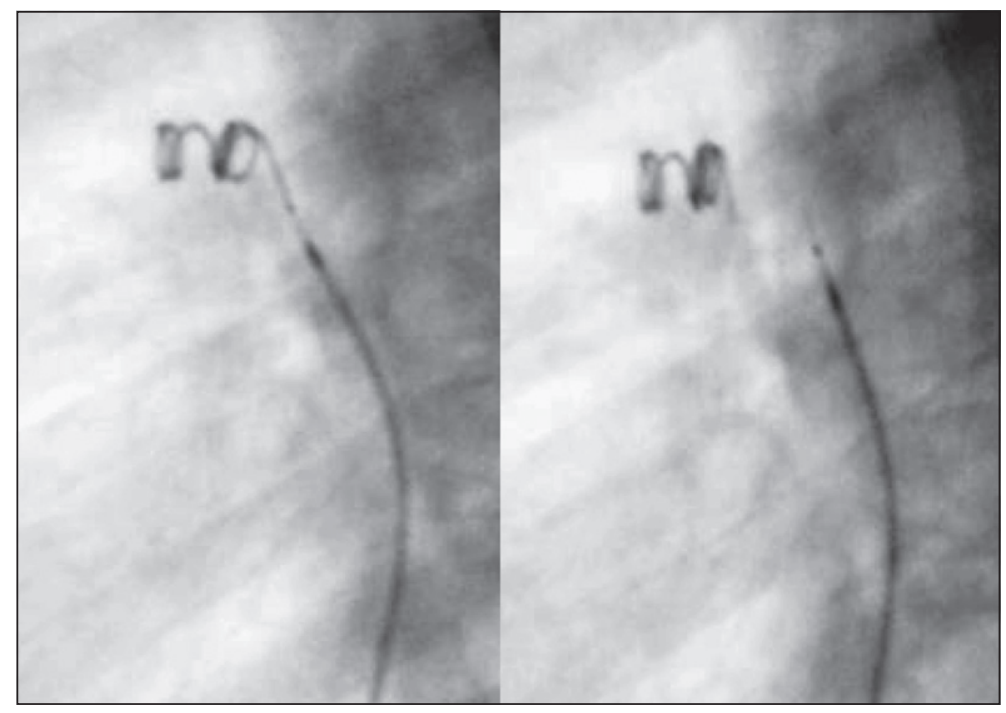

Figura 2. Imágenes de secuencia colocación del coil en el ductus arterioso, liberación del dispositivo. 
Tabla 1. Datos de las pacientes intervenidas vía transcáteter para cierre de Ductus Arterioso Persistente

\begin{tabular}{|c|c|c|c|c|c|c|}
\hline Características & Paciente 1 & Paciente 2 & Paciente 3 & Paciente 4 & Paciente 5 & Paciente 6 \\
\hline Edad & 12 años & 1 año 4 meses & 3 años 11 meses & 2 años & 2 meses & 4 años \\
\hline Peso & 34 kilos & 12,8 kilos & 16 kilos & 14 kilos & 5,5 kilos & 15 kilos \\
\hline Diámetro ECO & $1,5 / 2,5 \mathrm{~mm}$ & $2 / 2,5 \mathrm{~mm}$ & $2 / 3,3 \mathrm{~mm}$ & $2 / 3 \mathrm{~mm}$ & $1,5 / 3,5 \mathrm{~mm}$ & $2,2 / 3 \mathrm{~mm}$ \\
\hline Tipo ductus & Krichenko A1 & Krichenko A1 & Krichenko A2 & Krichenko A2 & Krichenko E & Krichenko A1 \\
\hline PAP media & $18 \mathrm{mmHg}$ & $12 \mathrm{mmHg}$ & $20 \mathrm{mmHg}$ & $14 \mathrm{mmHg}$ & $32 \mathrm{mmHg}$ & $16 \mathrm{mmHg}$ \\
\hline Anestesia & General & General & General & General & General & General \\
\hline Shunt residual & No & No & No & No & No & No \\
\hline $\begin{array}{l}\text { Control a los } 7 \text { días, al } \\
\text { mes y } 6 \text { meses }\end{array}$ & $\begin{array}{l}\text { Sin complica- } \\
\text { ciones }\end{array}$ & $\begin{array}{l}\text { Sin complica- } \\
\text { ciones }\end{array}$ & $\begin{array}{l}\text { Sin complica- } \\
\text { ciones }\end{array}$ & $\begin{array}{l}\text { Sin complica- } \\
\text { ciones }\end{array}$ & $\begin{array}{l}\text { Sin complica- } \\
\text { ciones }\end{array}$ & $\begin{array}{l}\text { Sin complica- } \\
\text { ciones }\end{array}$ \\
\hline
\end{tabular}

control y se les efectuó controles a la semana, mes y seis meses de la intervención.

\section{Protocolo anestésico}

Se realizó valoración preoperatoria y consentimiento informado anestésico a los padres. Durante el procedimiento se monitorizaron con electrocardiografía de dos derivaciones, saturometría continua, monitoreo de presión arterial no invasiva, temperatura esofágica más fonendo esofágico. Uso de Hot Line para mantener temperatura de infusiones. Anestesia general balanceada, inducción inhalatoria con SEVORANE, permeabilización venosa y fentanilo 2-4 ug/ $\mathrm{kg}$, vía aérea con máscara laríngea según peso, uso de relajante muscular Atracurio 0,4 mg/kg si pesa más de $30 \mathrm{~kg}$. Analgesia postoperatoria con Paracetamol.

\section{Resultados}

La edad de los pacientes fue entre los 2 meses y los 12 años, con pesos comprendidos entre los 5,5-34 kg y un promedio $16,2 \mathrm{~kg}$. Todas eran de sexo femenino. Clínicamente presentaban soplos, cuatro eran sistólico y dos en maquinaria. El ecocardiograma mostro dilatación de cavidades izquierdas y un diámetro DAP con rango 1,5-3,5 $\mathrm{mm}$. Las características de las pacientes intervenidas se muestran en la tabla 1. En las seis pacientes se logró cierre completo angiográfico ductal no habiendo shunt residual posterior al implante del dispositivo. El procedimiento duró un tiempo promedio de 55 min con un rango de 42-75 min, con un tiempo de fluoroscopia entre 10-22 min y un promedio de $14 \mathrm{~min}$. No se registraron complicaciones en el desarrollo de la intervención en ninguna de las pacientes. Fueron dadas de alta al día siguiente después de tomar radiografía de tórax y ecocardiograma B-D color donde se visualizó el dispostivo in sede no detectando shunt residual al ecocardiograma de control. En los controles a los 7 días, al mes y a los 6 meses de realizada la intervención no se han evidenciado complicaciones y los pacientes se encuentran en óptimas condiciones de salud y realizan vida normal.

\section{Discusión}

El cierre de DAP a través de catéter utilizando espirales es una técnica segura y efectiva, siendo el tratamiento de elección en los ductus de tamaño pequeño y en un importante porcentaje de los medianos, con excelente tasa de cierre y muy baja incidencia de complicaciones. Para los DAP moderados y grandes existe una amplia gama de dispositvos oclusores (tipo tapón) disponibles hoy en día, muy efectivos pero que nos enfrentan al dilema de su alto costo. En esta cardiopatía congénita, la indicación de cierre está dada por el riesgo de endocarditis infecciosa, cuadros bronquiales a repetición, pobre incremento pondoestatrual, insuficiencia cardíaca y el potencial riesgo a largo plazo de morir por complicaciones asociadas a la insuficiencia cardíaca o de desarrollar 
enfermedad vascular irreversible (síndrome de Eisenmenger $)^{3-8}$. Dentro de las complicaciones durante el procedimiento podemos mencionar cierre incompleto con cortocircuitos residuales, la embolización del dispositivo ya sea por colocación incorrecta de la espiral, o que esta se desprenda accidentalmente. En caso de ocurrir la embolización del espiral éste se puede recuperar por medio de un catéter lazo, forceps de recuperación vascular, siendo extraido a traves de la vaina. En los últimos años hay reportes de embolización al pulmón donde se decide abandonar el dispositvo ya que son tan pequeños no dejando secuelas ni repercusiones de tipo funcional siendo esto menos perjudicial que el daño que se puede causar al intentar recuperar el dispositivo. También se pueden producir lesiones relacionadas con el acceso vascular (hematomas) los que raramente dejan secuelas y en su mayoría ceden en algunos días o semanas. Otras complicaciones son turbulencia u obstrucción de estructuras vecinas al dispositivo y hemólisis intravascular ${ }^{5,6}$. Se puede presentar ausencia de pulso arterial femoral post procedimiento en especial en neonatos, lo que puede ser transitorio o definitivo. El hematoma retroperitoneal, complicación de muy baja incidencia, puede llegar a ser grave en ocasiones asociado a punción arterial alta.

Para nuestro grupo la técnica transcatéter para cierre DAP pequeño y mediano ha resultado ser una técnica de tratamiento eficaz, rápida y sin complicaciones para estos primeros casos, que ha permitido tratar a un grupo seleccionado de pacientes sin necesidad de derivación interregional para su manejo. Esto repercute en beneficios para las familias de nuestros pacientes debido al no traslado del grupo familiar a la capital, tales como disminuir los costos económicos, laborales y el ausentismo escolar durante los períodos de evaluación y del tratamiento de esta patología.

\section{Referencias}

1.- Anderson $R C$ : Causative factors underlying congenital heart mal- formations. I, patent ductus arte- riosus. Pediatr 1954; 14: 143-51.

2.- Heusser RF, Clavería RC, Trincado GC, Garay GF, Urcelay MG, Arnaiz GP: Cierre transcatéter de Ductus Arterioso Persistente con espirales de Gianturco. Rev Chil Pediatr 2001; 72 (6): 516-23.

3.- Becker RP: Tratamiento quirúrgico del Ductus Arterioso Persistente. Rev Chil Cardiol 2009; 28 (4): 401-2.

4.- Krichenko A, Benson LN, Burrows P, Möes CAF, McLaughlin P, Freedom RM: Angiographic classification of the isolated, persistently patent ductus arteriosus. Am J Cardiol 1989; 67: 877-80.

5.- Magee AG: Transcatheter coil occlusion of the arterial duct. Eur Heart J 2001; 22 (19): 1817-21.

6.- Campbell $M$ : Natural history of persistent ductus arteriosus. Br Heart J 1968; 30: 4-13.

7.- Somoza F, Marino B: Cardiopatías Congénitas, Cardiología Perinatal. 2007 Imprenta Don Bosco, Buenos Aires, Argentina.

8.- Maure D, et al: Cierre percutáneo del Ductus arterioso con coils, diagnóstico y seguimiento ecocardiográfico a largo plazo. Rev Chil Ultrasonog 2009; 12: 45-50. 